\title{
MASS AMBIGUITIES IN CASCADE DECAYS
}

\author{
B. K. GJELSTEN \\ Laboratory for High Energy Physics, University of Bern, CH-3012 Bern, Switzerland \\ D. J. MILLER \\ Department of Physics and Astronomy, University of Glasgow, Glasgow G12 8QQ, U.K. \\ P. OSLAND \\ Department of Physics and Technology, University of Bergen, N-5020 Bergen, Norway \\ A. R. RAKLEV \\ Theory Division, Physics Department, CERN, CH-1211 Genève, Switzerland
}

\begin{abstract}
We review the use of invariant mass distributions in cascade decays to measure the masses of New Physics (NP) particles in scenarios where the final NP cascade particle is invisible. We extend earlier work by exploring further the problem of multiple solutions for the masses.

Keywords: SUSY; BSM; MSSM.
\end{abstract}

\section{Introduction}

Inspired by the precise determination of the dark matter relic density made possible with data from the WMAP satellite ${ }^{1,2,3}$ New Physics (NP) models with a weakly interacting massive particle have flourished over the last years. Such particles, whether they appear in models of supersymmetry, universal extra dimensions or little Higgs theories, are invisible to the detectors of collider experiments, and to be viable dark matter candidates, certainly stable on the scale of the current age of the universe. The conservation of a NP quantum number, be it R-parity, KKparity or T-parity, ensures a cascade decay of all heavier NP particles into the lightest NP particle. Measuring NP particle masses in such scenarios by reconstructing mass peaks is then in general no longer possible.

The well studied alternative to this is to use measurements of the endpoints of invariant mass distributions. ${ }^{4,5,6,7,8,9,10}$ As we can express endpoint positions in terms of the NP masses, we can in principle solve for the masses if we have at least as many endpoints as unknown masses. However, there are nontrivial problems with this method. Generally the expressions for the endpoints are complicated functions, leading to possible multiple solutions, and strong correlations between the measured masses. ${ }^{11}$ As an illustration of this problem, we consider the decay chain

$$
\tilde{q}_{L} \rightarrow \tilde{\chi}_{2}^{0} q \rightarrow \tilde{\ell}_{R} \ell_{n} q \rightarrow \tilde{\chi}_{1}^{0} \ell_{f} \ell_{n} q
$$

and show in Fig. 1 the quantity

$$
\mu=\sum_{i}\left|m_{i}^{\text {false }}-m_{i}^{\text {true }}\right| / m_{i}^{\text {true }}
$$

for a scan over the SPS1a ${ }^{12}$ mSUGRA scalar $\left(m_{0}\right)$ and gaugino $\left(m_{1 / 2}\right)$ mass plane for a trilinear coupling $A_{0}=-m_{0}, \tan \beta=10$ and $\mu>0$. The sum in (2) runs over the squark, slepton and neutralino masses in the decay chain (1).

We find that for virtually all points in the mass plane where the decay chain (1) exists, and where the lightest supersymmetric particle (LSP) is not the stau (at low $m_{0}$ ), we 


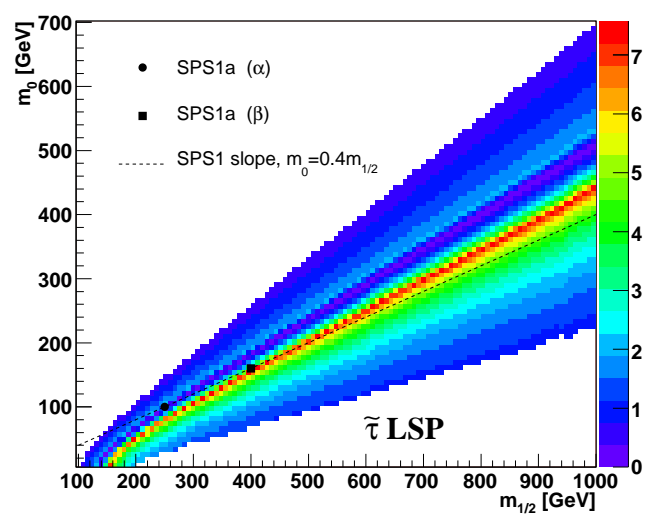

Fig. 1. Scan of $\mu$, as given by Eq. (2), over the SPS1a mass plane. In the top white area the decay chain (1) is not kinematically accessible, while the bottom white area has a charged stau LSP.

get a false solution from the set of endpoints. Along the middle of the physical wedge in this plane, and intersecting the SPS1a line, the above quantity can become rather large. In an experiment one might also encounter the problem of "feet", i.e. structures in the distributions near the endpoints that are hidden by background. ${ }^{8,13}$

We have previously started an investigation into the possible improvements to this method that can result from fitting the whole invariant mass distribution to an analytical expression derived for its shape. ${ }^{13}$ We found that knowledge of the shape will predict any possible irregular features at the endpoints for a measured set of masses, thus essentially removing the problem of feet. Here we want to investigate further what effect knowledge of the shape of the invariant mass distribution has on the problem of multiple solutions.

\section{Multiple solutions}

Using as our example the four possible ${ }^{\mathrm{a}}$ invariant mass distributions for the SUSY de-

\footnotetext{
${ }^{\mathrm{a}}$ We can form the invariant mass of the two leptons, the two leptons and a jet, but assuming that we cannot, in general, identify which lepton is the lepton nearest to the squark in the decay chain, we use jet-
}

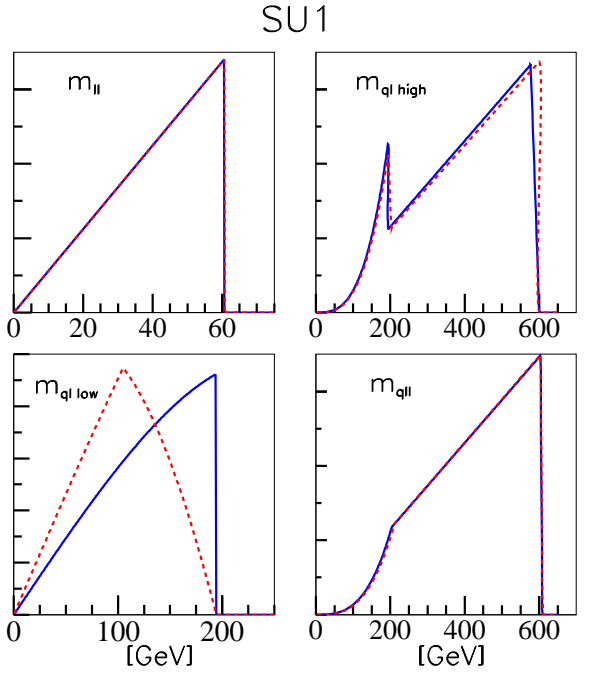

Fig. 2. Invariant mass distributions for the SU1 benchmark point and the decay chain (1) with arbitrary normalisation. In blue (solid) are the distributions for the nominal masses, in red (dashed) are the distributions for the masses in the false solution.

cay chain (1) we show in Fig. 2 the shape of these distributions, as found in Ref. 13, for the nominal masses of the ATLAS SU1 staucoannihilation benchmark point. (One may construct other invariant mass distribution by imposing cuts, but for simplicity we will neglect them here.)

For SU1 there is also another set of masses that give the same endpoints for the invariant mass distributions. If only endpoints are measured we have two indistinguishable solutions for the SUSY masses. The two sets are given in Table 1 as the nominal masses of the benchmark point, and the false solution from the nominal endpoints of the benchmark point.

In Fig. 2 we see that there are only small visible differences in the shapes between the two mass sets of SU1 for three of the distributions, but for $m_{q l(\text { low })}$ there is a marked

lepton combinations that give the highest and lowest invariant mass. 
Table 1. Nominal and false sets of masses for benchmark points $[\mathrm{GeV}]$.

\begin{tabular}{lrrrr}
\hline Set & $m_{\tilde{\chi}_{1}^{0}}$ & $m_{\tilde{l}_{R}}$ & $m_{\tilde{\chi}_{2}^{0}}$ & $m_{\tilde{q}_{L}}$ \\
\hline SU1 nom. & 137 & 254 & 264 & 760 \\
SU1 false & 122 & 127 & 246 & 744 \\
SU3 nom. & 118 & 155 & 219 & 631 \\
SU3 false & 347 & 411 & 452 & 900 \\
\hline
\end{tabular}

change. Similar differences in the shapes can also be seen in three of the distributions for SU3, the bulk-region benchmark point, shown in Fig. 3, although they are individually somewhat less distinct than for SU1. For both cases it seems likely that the false solution could be ruled out, with the expected high statistics at the LHC for SUSY scenarios with sub-TeV squark masses. For the SU3 benchmark point the large differences in mass between the solutions should also show up clearly in the measured cross sections.

This is unfortunately not the situation for all models. For the SPS1a bulk-region benchmark point ${ }^{12}$ the differences in the shapes are very small. After adding smear-

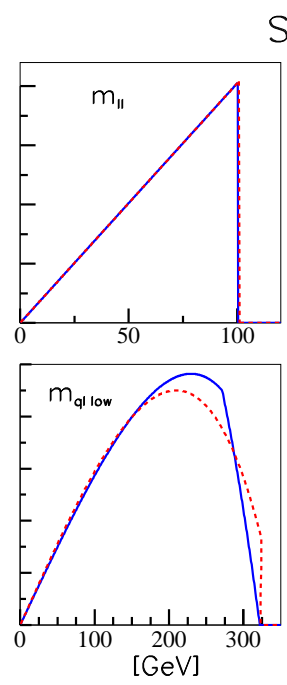

\section{SU3}

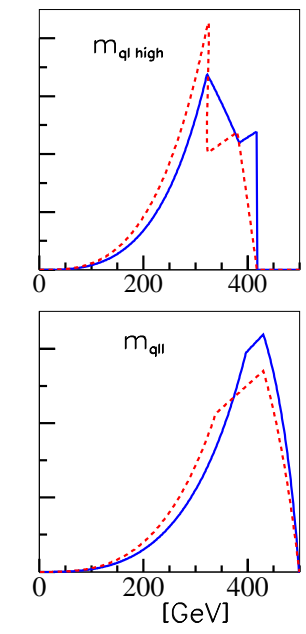

Fig. 3. Invariant mass distributions for the SU3 benchmark point. See caption of Fig. 2 for details. ing from detector effects, combinatorics from picking the correct jet, initial and final state radiation etc., separating the two mass sets for SPS1a will be very difficult and require very large statistics and an extremely good understanding of the detector, if at all possible.

It is then natural to ask how effective the shape can be in distinguishing multiple solutions over a wide range of SUSY models. In Fig. 4 we show a scan over the SPS1a mass plane. We plot the value of

$$
D=\frac{1}{2 N} \sum_{i=1}^{N} \int_{0}^{m_{i}^{\max }}\left|f_{i}(m)-g_{i}(m)\right| d m
$$

where $f_{i}$ and $g_{i}$ are the invariant mass distributions for the false and nominal sets of mass solutions (normalized to $\int d m f(m)=1$, $\left.\int d m g(m)=1\right)$, with the $i$-index running over the number of distributions $N$ (here $N=4$ ), and where $m_{i}^{\max }$ is the endpoint of the $i$-th distribution. The numerical prefactor ensures that $D \leq 1$.

The difference in shape between the nominal and false solutions, as quantified by the $D$-value is fairly constant at around $D \approx 0.07$, except for a wedge in the mass plane, where we have a transition between

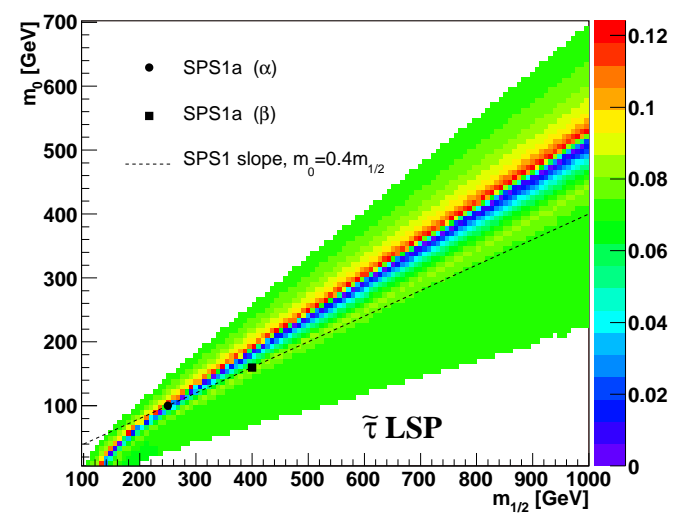

Fig. 4. Scan of $D$, as given by Eq. (3), over the SPS1a mass plane. In the top white area the decay chain (1) is not kinematically accessible, while the bottom white area has a charged stau LSP. 
different forms for the analytical expressions for the $m_{q l(\text { low })}$ and $m_{q l \text { (high) }}$ distributions. ${ }^{13}$ In this region the $D$-value changes rapidly from low to high values, and from Fig. 4 we see that the SPS1a benchmark point suffers from an unfortunate position, giving it a value of $D=0.016$. Comparing to Fig. 1 we find that while large mass differences can give large differences in shape, there are certainly areas where the correct solution could be readily identified from the shape, even when the mass differences are small, and thus where a comparison of cross sections would be difficult.

Due to smearing from the experimental effects mentioned above, it is difficult to calculate exactly for what $D$-value two sets of solutions can be distinguished for some given integrated luminosity at the LHC. However, assuming that the two distributions of SU1 are distinguishable, and using as a basis for comparison that we find a $D$-value of $D=$ 0.070 for SU1, this suggests that we should be able to distinguish between the nominal and false solutions in most of the parameter space shown in Fig. 4. We have performed such scans for different values of $A_{0}$ and $\tan \beta$, in the mass planes of the SPS1b, SPS3 and SPS5 benchmark points, ${ }^{12}$ and find similar results there.

\section{Conclusions}

We have discussed aspects of the use of analytical expressions for invariant mass distributions in determining masses in NP scenarios, using a well studied SUSY decay chain as our example. We have focused on the possibility of using differences in the shapes of distributions to remove false solutions that appear if only the endpoints of the invariant mass distributions are used to determine the NP masses. Our results from scans of the mSUGRA parameter space indicate that with the exception of certain limited areas, the shapes of the distributions should in general be well suited to reject the false solu- tion, given that enough statistics are available, and may be applied even when a comparison of cross sections would be difficult.

\section{Acknowledgments}

ARR acknowledges support from the European Community through a Marie Curie Fellowship for Early Stage Research Training. This research has been supported in part by the Research Council of Norway and the Swiss National Science Foundation.

\section{References}

1. C. L. Bennett et al., Astrophys. J. Suppl. 148, 1 (2003) [arXiv:astro-ph/0302207].

2. D. N. Spergel et al., Astrophys. J. Suppl. 148, 175 (2003) [arXiv:astro-ph/0302209].

3. D. N. Spergel et al., arXiv:astro-ph/ 0603449.

4. I. Hinchliffe, F. E. Paige, M. D. Shapiro, J. Soderqvist and W. Yao, Phys. Rev. D55, 5520 (1997) [arXiv:hep-ph/9610544].

5. H. Bachacou, I. Hinchliffe and F. E. Paige, Phys. Rev. D62, 015009 (2000) [arXiv:hep$\mathrm{ph} / 9907518$.

6. B. C. Allanach, C. G. Lester, M. A. Parker and B. R. Webber, JHEP 0009, 004 (2000) [arXiv:hep-ph/0007009].

7. C. G. Lester, CERN-THESIS-2004-003.

8. B. K. Gjelsten, D. J. Miller and P. Osland, JHEP 0412, 003 (2004) [arXiv:hep-ph/ 0410303].

9. G. Weiglein et al. [LHC/LC Study Group], Phys. Rept. 426, 47 (2006) [arXiv:hep$\mathrm{ph} / 0410364]$

10. B. K. Gjelsten, D. J. Miller and P. Osland, JHEP 0506, 015 (2005) [arXiv:hep-ph/ 0501033].

11. B. K. Gjelsten, D. J. Miller and P. Osland, arXiv:hep-ph/0507232; arXiv:hep-ph/0511008.

12. B. C. Allanach et al., in Proc. of the APS/DPF/DPB Summer Study on the Future of Particle Physics (Snowmass 2001), ed. N. Graf, Eur. Phys. J. C25, 113 (2002) [arXiv:hep-ph/0202233].

13. D. J. Miller, P. Osland and A. R. Raklev, JHEP 0603, 034 (2006) [arXiv:hep$\mathrm{ph} / 0510356]$. 